\title{
Effect of Asymmetric Cold Rolling on Texture of a Commercially Pure Copper
}

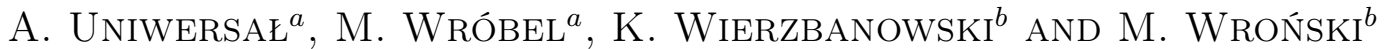 \\ ${ }^{a}$ Faculty of Metals Engineering and Industrial Computer Science, AGH University of Science and Technology, \\ al. A. Mickiewicza 30, 30-059 Kraków, Poland \\ ${ }^{b}$ Faculty of Physics and Applied Computer Science, AGH University of Science and Technology, \\ al. A. Mickiewicza 30, 30-059 Kraków, Poland
}

\begin{abstract}
This study deals with the influence of the rolling asymmetry and applied strain on the crystallographic texture of polycrystalline copper. It was shown that textures of asymmetrically rolled materials are rotated with respect to those rolled symmetrically and the rotation angle increases both with the rolling asymmetry and strain. The asymmetric rolling leads also to a decrease of intensity of texture components forming skeleton lines in the orientation space. However, individual texture components exhibit different sensitivity to the effect of asymmetric rolling.
\end{abstract}

DOI: 10.12693/APhysPolA.130.1049

PACS/topics: 81.40.Ef

\section{Introduction}

The rolling process, commonly used for the flat products manufacture (i.e., plates and sheets) has two symmetry planes/two twofold axes. One symmetry plane is perpendicular to the normal direction (ND) and the second one - to the transverse direction (TD) of the rolled sample. Consequently, such process is called symmetric rolling (SR) and its products have the orthotropic symmetry. This symmetry is reflected in crystallographic textures [1]. On the other hand, rolling process without such the symmetry is recently developed as it can improve both efficiency of technology and properties of rolled products [1-8]. Such a rolling process, called asymmetric rolling (AR), has only one symmetry plane (perpendicular to TD) and its products reveal the monoclinic symmetry. Generally, the AR texture is close to the SR one, but is rotated around TD [1-15]. Such the texture effect of AR was recently found, even for a relatively small strains and rolling asymmetries, despite a high crystal symmetry of the deformed crystals (i.e., copper with the face-centered cubic (fcc) structure and the symmetry class $F m 3 m$ ) [1]. The observed texture modifications can be reproduced by crystalline models of plastic deformation [16-18]. In our previous work [1], the rolling asymmetry resulted from a difference in angular velocities of the same rolls. However, at low deformation it was not possible to display other possible texture modifications appearing besides its rotation around TD. The present paper is focused on this problem. It is also interesting to test whether a similar texture rotation can be obtained for another way of inducing the rolling asymmetry.

\section{Experimental procedure}

Samples of commercially pure copper, of $5 \times 50 \times$ $200 \mathrm{~mm}^{3}$ size, were annealed during $1.5 \mathrm{~h}$ at $450^{\circ} \mathrm{C}$ and than deformed at room temperature by SR and AR up to $80 \%$ thickness reduction. This deformation was imposed in several passes, but for each sample the intermediary deformation scheme was the same. The rolling process was performed using the reversible quarto rolling mill. The rolling asymmetry was caused by different diameters of the rolls. Thus, the rolling asymmetry coefficient, $A$, was defined as:

$$
A=\frac{D_{1}}{D_{2}}
$$

where $D_{1}$ and $D_{2}$ are diameters of the lower and upper rolls, respectively. The diameter of the lower work roll was always higher and the following values of the asymmetry ratio $(A)$ were used: $1.0,1.1,1.15,1.0,1.25$, and 1.3 .

The $\{111\},\{200\},\{220\}$, and $\{311\}$ incomplete pole figures were measured on the X-ray Empyrean diffractometer from PANalytical Co. The parallel beam of $\mathrm{Cu} K_{\alpha}$ radiation was used. The measurements were performed on the sample surface parallel to the rolling plane and located in the middle of the rolled bar thickness. The exposed surfaces were gently polished and etched before X-ray measurements. The experimental pole figures were used next to calculate the orientation distribution function (ODF) [19] from which, in turn, complete pole figures were recalculated and skeleton lines of the texture were determined. $\mathrm{ODF}(\mathrm{g})$ is the probability density function, defined in the orientation space (OS), and it estimates a volume fraction of grains, $\mathrm{d} V / V$, with crystallographic orientations between $g$ and $g+\mathrm{d} g$. LaboTex [20] commercial software was used for its calculation. In the present paper, the Bunge convention [19] of the Euler angles $\left(\phi_{1}, \Phi\right.$ and $\left.\phi_{2}\right)$ was used, therefore a crystal orientation $g$ is determined by a set of these three angles. In Table I, the Euler angles and the Miller indices of texture components used in this paper are collected.

\section{Results and discussion}

The initial material has a relatively weak texture (Fig. 1a). However, some components of texture can be noted, i.e., $B, C$, and $W$ orientations. 
TABLE I

Ideal texture components for rolled fcc metallic materials.

\begin{tabular}{c|c|c|c|c}
\hline \hline Name & \multicolumn{2}{|c|}{ Symbol } & Miller indices & $\begin{array}{c}\text { Euler angles }\left[^{\circ}\right] \\
\phi_{1}, \Phi, \phi_{2}\end{array}$ \\
\hline Goss & $G$ & $\diamond$ & $\{110\}\langle 001\rangle$ & $0,45,0$ \\
Brass & $B$ & $\bigcirc$ & $\{110\}\langle 112\rangle$ & $35,45,0$ \\
Copper & $C$ & $\triangle$ & $\{112\}\langle 111\rangle$ & $90,35,45$ \\
Taylor & $T$ & $\square$ & $\{4411\}\langle 11118\rangle$ & $90,27,45$ \\
S1 & $S_{1}$ & $\square$ & $\{124\}\langle 211\rangle$ & $59,29,63$ \\
S2 & $S_{2}$ & $\square$ & $\{123\}\langle 421\rangle$ & $47,37,63$ \\
S3 & $S_{3}$ & $\nabla$ & $\{123\}\langle 634\rangle$ & $59,37,43$ \\
Cube & $W$ & $\square$ & $\{100\}\langle 001\rangle$ & $0,0,0$
\end{tabular}
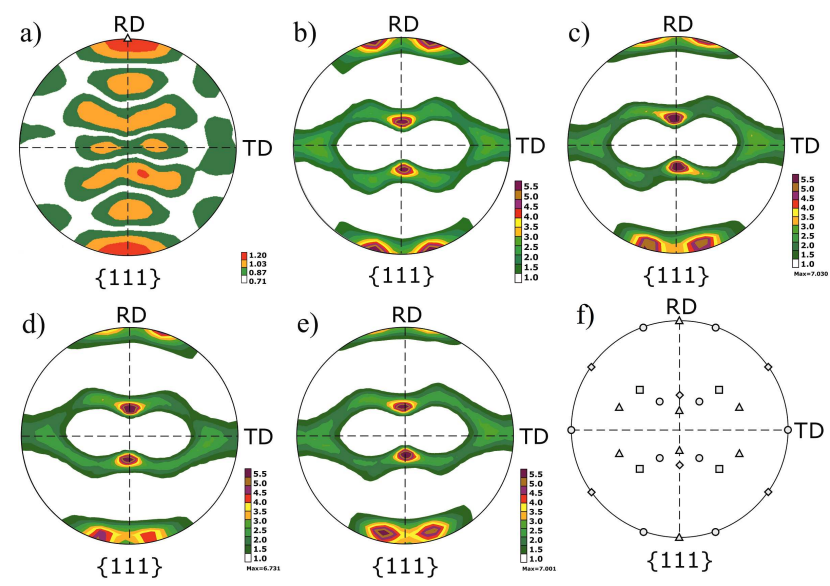

Fig. 1. $\{111\}$ pole figures: for: the initial material (a), after SR with $A=1.0$ (b), after AR with $A=1.1$ (c), after AR with $A=1.2(\mathrm{~d})$, after AR with $A=1.3(\mathrm{e})$. The samples were rolled to $80 \%$ reduction in thickness and positions of main texture components for SR material are shown in $(\mathrm{f})$.

Textures of deformed material (Fig. 1b-e) are well developed and typical for the cold rolled copper. So, components characteristic for this texture are marked, i.e., $B, C, S$, and $G$. Positions of these components on the $\{111\}$ pole figure are shown in Fig. 1f. The texture of SR material shows almost perfect orthorhombic symmetry, despite the fact that any sample symmetry was assumed during calculation. Thus, ND, rolling direction (RD), and TD are twofold axes of pole figures and the texture components are located in OS very close to typical positions of the ideal orientations from Table I. However, in the case of AR material, texture components are rotated from their typical positions and the symmetry characteristic for SR is not observed. Textures of the AR material are rotated in one sense around TD comparing with texture of the SR material, as was expected. So, one can conclude that TD-rotation of the rolling texture depends on the rolling asymmetry but not on the way in which this asymmetry was induced (different roll diameters instead of different angular velocities). The rotation angle increases with the rolling asymmetry (Fig. 2). Moreover, data collected in Fig. 2b shows that the angle of rotation increases significantly with strain. Thus, texture rotation may be associated with different stress state during AR process, as compared with the SR one. Indeed, we confirmed that an increased amplitude of the shear stress, $\Sigma_{\mathrm{RD}, \mathrm{ND}}$, appears during AR. However, a detailed analysis of this aspect is beyond the scope of the present paper.
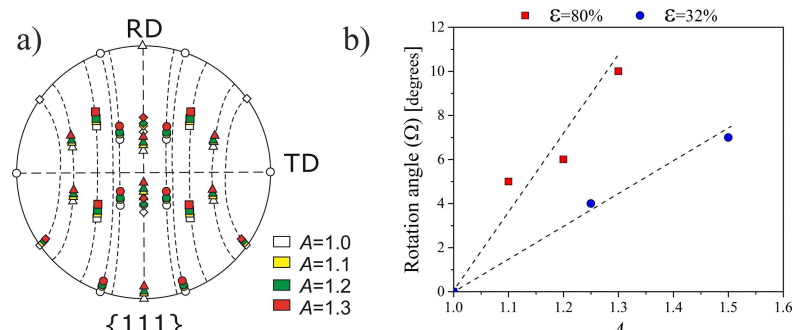

Fig. 2. Positions of texture components for the symmetrically $(A=1.0)$ and asymmetrically $(A=1.1,1.2$, 1.3 ) rolled material (a), and effect of the rolling asymmetry and strain (expressed as reduction of thickness) on the angle of texture rotation around TD (b); data for $32 \%$ reduction was taken from [1].

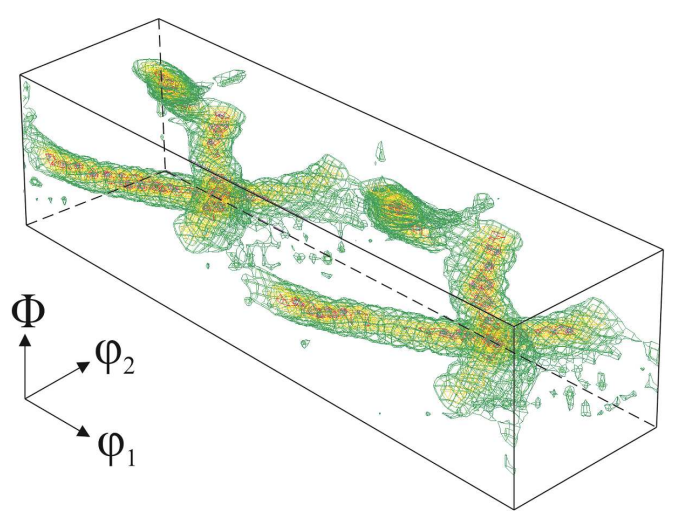

Fig. 3. Texture of the AR material $(A=1.3)$ shown in the orientation space. $\phi_{1}$ varies in the range $0^{\circ}-180^{\circ}, \Phi$ and $\phi_{2}$ - in the range $0^{\circ}-90^{\circ}$.

Rolling copper textures form a branch in OS (Fig. 3). However, in the three-dimensional OS some details of textures are not always clearly seen. Therefore, fibers (i.e., lines in OS which connect main texture orientations) will be useful in texture description. Two fibers are typically developed in fcc rolling textures, i.e., the $\alpha$-fiber and the $\beta$-fiber [20]. The $\alpha$-fiber runs from $G$ orientation to $B$ orientation. The $\beta$-fiber connects $C, S$, and $B$ orientations and usually this fiber is prominent in copper samples heavily deformed by rolling.

However, the ideal fibers were not developed, because positions of the ODF maxima, especially in AR textures, may be shifted from ideal orientations listed in Table I. Therefore, we examine the lines, which join ODF maxima close to ideal orientations, and we call them skeleton lines (SL). In the present study SLs can be considered as distorted $\alpha$ - and $\beta$-fibers and we call them as $\alpha$-fiber SL 
and $\beta$-fiber SL, respectively. It should be mentioned that texture analysis based on skeleton lines is frequently used in the literature [19, 21-28]. The effect of rolling asymmetry on SLs is shown in Figs. 4 and 5. The rolling asymmetry causes shifts of the $\alpha$-fiber SL in OS along $\Phi$ axes and reduces their ODF intensities by around $20 \%$ (Figs. 4a,b). It is visible on the example of $G$ and $B$ components. On the other hand, a clear effect of the rolling asymmetry can be observed on the $\beta$-fiber SL and it consists in a significant decrease of intensity of all texture components from this SL (Fig. 4c). It is observed already for $A=1.1$ and the effect is more marked for higher values of the asymmetry ratio $A$. a)

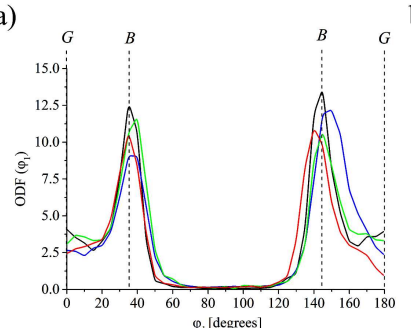

c)

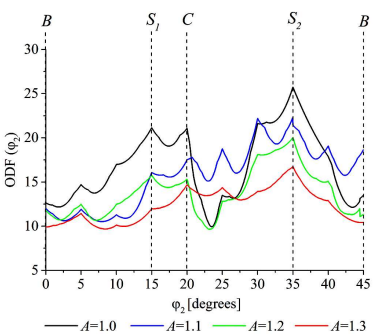

b)
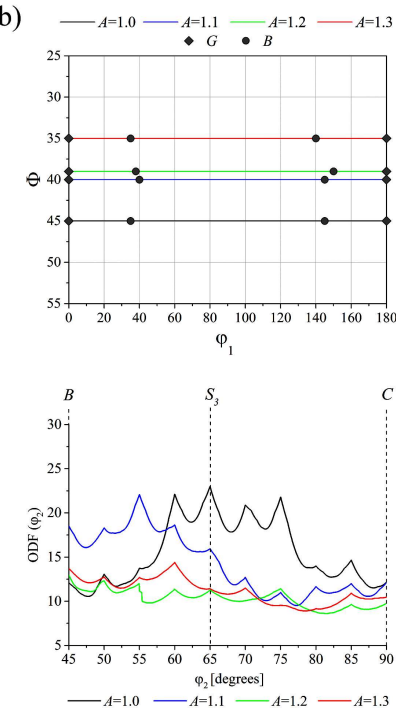

Fig. 4. Effect of rolling asymmetry on: intensity of $\alpha$ fiber SL (a), position of $\alpha$-fiber SL (b), intensity of $\beta$ fiber SL (c). a)
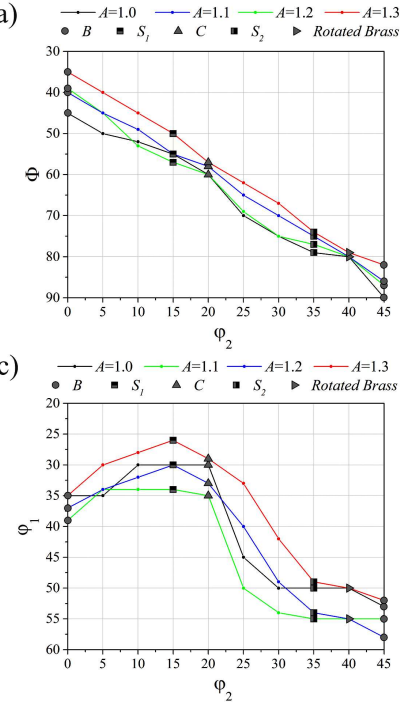

c) $\rightarrow A=1.0 \rightarrow A=1.1 \rightarrow{ }^{2} \rightarrow 1.2 \rightarrow{ }_{A}=1.3$

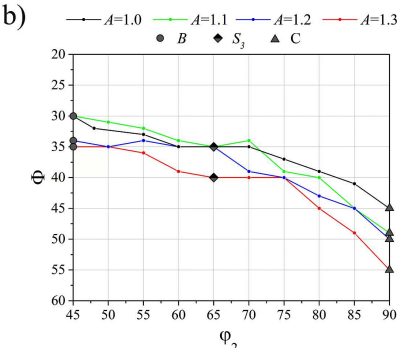

d)

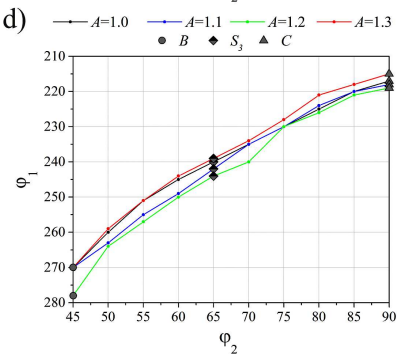

Fig. 5. Effect of rolling asymmetry on positions of $\beta$ fiber SL in the OS: in $\Phi$ vs. $\phi_{2}$ plot $(\mathrm{a}, \mathrm{b})$, and in $\phi_{1}$ vs. $\phi_{2}$ plot $(\mathrm{c}, \mathrm{d})$.
The strongest effect of asymmetry on intensity reduction refers to $S$ orientations. The effect of $\mathrm{AR}$ on the position of $\beta$-fiber SL is shown in Fig. 5. A relatively large displacement of $B$ component along $\Phi$ and $\phi_{1}$ axes can be seen. The same concerns $S_{1}$ and $S_{2}$ components. On the other hand, the shift of $C$ component is higher along $\phi_{1}$ than along $\Phi$ axis for $\phi_{2}=20^{\circ}$, but the inverse relation is observed for $\phi_{2}=90^{\circ}$. The component in position $\phi_{2}=40^{\circ}$ and $\Phi=80^{\circ}$ (called rotated Brass) is shifted only along $\phi_{1}$ axis. In Refs. [29-32] it was shown that $B$ orientation has a relatively wide range of stability for the stress state close to the Tucker type stress tensor [33, 34] (i.e., the typical stress state for SR: $\Sigma_{\mathrm{RD}-\mathrm{RD}}=-\Sigma_{\mathrm{ND}-\mathrm{ND}}$ ), so a relatively important rotation of this orientation indicates a significant change of the stress state during AR.

\section{Conclusions}

The present research showed that asymmetric rolling textures are rotated around the transverse direction, comparing to the texture developed during symmetric rolling. The rotation angle increases both with the asymmetry ratio and the amount of deformation and does not depend on the way in which this asymmetry is realized. It was also found that rolling asymmetry leads to complicated shifts of the $\beta$-fiber skeleton line in the orientation space and to a significant decrease of texture component intensities.

\section{Acknowledgments}

Financial support of the National Science Centre (Poland), decision DEC 2013/09/N/ST8/04164, is appreciated.

\section{References}

[1] A. Uniwersał, M. Wróbel, S. Wroński, I. KalembaRec, M. Wroński, K. Wierzbanowski, A. Baczmański, B. Bacroix, Arch. Metall. Mater. 61, (2016).

[2] E.A. Maksimov, Metallurgist 54, 753 (2011).

[3] P.P. Gudur, M.A. Salunkhe, U.S. Dixit, Int. J. Mech. Sci. 50, 315 (2008).

[4] Y. Pang, Y. Hu, Q. Sun, Ch. Liu, Adv. Mater. Res. 97-101, 597 (2010).

[5] F. Farthatnia, M. Salimi, Int. J. Eng. Sci. Technol. 3, 147 (2011).

[6] S.A.A. Akbari Mousavi, S.M. Ebrahimi, R. Madoliat, J. Mater. Process. Tech. 187-188, 725 (2007).

[7] J.-S. Lu, O.-K. Harrer, W. Schwenzfeier, F.D. Fisher, Int. J. Mech. Sci. 42, 49 (2000).

[8] A. Kawałek, Achiev. Mater. Manufact. Eng. 23, 63 (2007).

[9] M. Wroński, K. Wierzbanowski, S. Wroński, B. Bacroix, M. Wróbel, A. Uniwersał, Mater. Sci. Eng. 82, 012074 (2015).

[10] M. Wroński, K. Wierzbanowski, A. Baczmanski, S. Wroński, B. Bacroix, M. Wróbel, A. Lodini, Adv. Mater. Res. 996, 688 (2014).

[11] M. Wroński, K. Wierzbanowski, S. Wroński, B. Bacroix, Met. Mater. Int. 21, 805 (2015). 
[12] S. Wroński, K. Wierzbanowski, B. Bacroix, Ch. Thierry, M. Wróbel, J. Cent. South Univ. T. 20, 1443 (2013).

[13] S. Wroński, K. Wierzbanowski, B. Bacroix, M. Wróbel, Mater. Sci. Forum 638-642, 2811 (2010).

[14] M. Wroński, K. Wierzbanowski, L. Pytlik, B. Bacroix, M. Wróbel, A. Baczmański, A. Lodini, Mater. Sci. Forum 777, 1 (2014).

[15] S. Wroński, K. Wierzbanowski, B. Bacroix, T. Chauveau, M. Wróbel, A. Rauch, F. Montheillet, M. Wroński, Arch. Metall. Mater. 54, 89 (2009).

[16] K. Wierzbanowski, A. Baczmanski, P. Lipinski, A. Lodini, Arch. Metall. Mater. 52, 77 (2007).

[17] A. Baczmański, A. Tidu, P. Lipinski, M. Humbert, K. Wierzbanowski, Mater. Sci. Forum 524-525, 235 (2006).

[18] A. Baczmański, K. Wierzbanowski, J. Tarasiuk, M. Ceretti, A. Lodini, Rev. Metall. - Paris 94, 1467 (1997)

[19] H.J. Bunge, Texture Analysis in Materials Science, Butterworths, London 1982.

[20] LaboTex, Software for Texture Analysis, Labosoft s.c., Kraków 2014.

[21] O. Engler, V. Randle, Introduction to Texture Analysis, Macrotexture, Microtexture and Orientation Mapping, 2nd ed., CRC Press, Boca Raton 2010, p. 3.
[22] H.J. Bunge, in: Preferred Orientation in Deformed Metal and Rocks: An Introduction to Modern Texture Analysis, Ed. H.-R. Wenk, Academic Press, London 1985, p. 107.

[23] A.D. Rollett, S.I. Wright, in: Texture and Anisotropy, Preferred Orientations in Polycrystals and their Effect on Materials Properties, Eds. U.F. Kocks et al., Cambridge University Press, 2000, p. 185.

[24] J. Hirsch, K. Lucke, Acta Metall. 36, 2863 (1988).

[25] J. Hirsch, K. Lucke, Acta Metall. 36, 2905 (1988).

[26] T. Leffers, R.K. Ray, Prog. Mater. Sci. 54, 351 (2009).

[27] W. Olek, J.T. Bonarski, BioResources 7, 1729 (2012).

[28] M. Wróbel, J. Pospiech, J. Bonarski, M. Blicharski, S. Dymek, Archiv. Metall. 47, 185 (2002).

[29] K. Wierzbanowski, A. Clement, Cryst. Res. Technol. 19, 202 (1984).

[30] M. Wróbel, W. Łatas, Archiv. Metall. 40, 333 (1995).

[31] M. Wróbel, S. Dymek, M. Blicharski, Archiv. Metall. 35, 245 (1990).

[32] M. Wróbel, S. Dymek, M. Blicharski, Zeit. Metallkd. 85, 415 (1994).

[33] G.E.G. Tucker, Acta Metall. 12, 1093 (1964).

[34] M. Wróbel, Ph.D. Thesis, AGH, Kraków 1989. 